stony meteorites originated in a primary object of roughly lunar size formed about 4,000 million years ago. There are two contributions concerned mainly with space-research techniques : H. Friedman on the rocket astronomy he has done so much to promote, and L. Goldberg on astronomy from satellites. Finally, J. W. Townsend outlines a programme for future space research, and $R$. Jastrow describes the Earth's outer atmosphere.

The book can be thoroughly recommended, and fully justifies the publishers' claim that it is a "comprehensive survey of the most recent developments in space science", though the developments are now not quite so recent as they were, since the increased pace of scientific progress has not been matched by any corresponding acceleration in printing, proof-reading -or reviewing. All but one of the papers given at the symposium were first printed in the November 1959 issue of the Journal of Geophysical Research, and the book is a faithful reproduction of the pages from the Journal, including all the misprints. 'The book illustrates a growing and deplorable tendency to reprint unchanged, in book form, scientific articles which are known to have misprints. If publishers wish to idopt this practice for economy, they should surely include a list of errata : for this book, such a list could well be headed by the value for the age of the Moon on page 143, which is in error by a factor of $10^{18}$.

D. G. King-Helf

\section{INVARIANCE PROPERTIES OF PARTICLES AND FIELDS}

Theory of Elementary Particles

By Paul Roman. (Series in Physics.) Pp. xii +575. (Amsterdam: North-Holland Publishing Company; New York: Interscience Publishers, Inc., 1960.) $100 s$.

THE progress that has been made in establishing a basic theory of the elementary particles and their interactions has depended to a large extent on the use of invariance properties under transformations. These range from the obvious Lorentz transformations to rotations and reflexions in isobaric space. They give rise to conservation laws, selection rules and relationships between reactions, all of which can be tested by experiment. Their deduction does not necessitate the solution of the field equations, which has so far not proved to be generally possible for the strong interactions. For the weak interactions almost complete understanding has been achieved as a result of the comparatively recent discovery of the non-conservation of $P$ and $C$.

Dr. Roman's book is particularly concerned with the invariance properties and their influence on the structure of the quantum field theory used to describe the elementary particles and their interactions. The treatment is outstandingly good. It is inevitably mathematical and requires an effort on the part of the reader (particularly the experimental variety), as is recognized by the author in his preface. He has most successfully sugared the pill by setting out the mathematics in considerable detail and carefully discussing its implications and limitations. The experimental implications are kept constantly in view and it is made clear how experimental results have determined the structure of the theory at every stage.
The first 100 pages are devoted to the theory of the four-dimensional orthogonal group and its representations. In the next 114 pages the quantum field theory is developed, starting with a classical treatment of the field equations to establish their form for the different fields. Quantization of the fields is then introduced, leading to the commutation rules and the use of creation and annihilation operators. The remainder of the book is devoted to the invariance properties. In particular, $P, C, T$ transformations and the relation between them are considered in detail and a section is devoted to the effects of the non-conservation of $P$ and $C$ in weak interactions. Transformations in isobaric space are then introduced, leading to the Gell-Mann Nishijima classification of the elementary particles. There are interesting discussions of the $|\Delta t|=\frac{1}{2}$ selection rule and of the properties of neutral $K$ mesons. Finally, more complete mathematical models of the elementary particles are considered, including the Prentkid'Espagnat theory of isospace. It should perhaps be mentioned that no attempt is made to include the methods (such as dispersion relations) that are being tried for dealing with the strong interactions.

This book can be recommended unhesitatingly to those who wish to become familiar with the basic theory of the elementary particles and their interactions. It will also be a vital source of reference for those who are only intermittently concerned with the basic aspects of the theory.

\section{A. Ashmore}

\section{CHEMICALS AND INSECTS}

Methods of Testing Chemica's on Insects, Vol. 2. Edited by Harold H. Shepard. Pp. iii + 248. (Minneapolis, Minn.: Burgess Publishing Company; London: Mayflower Publishing Co., Ltd., 1960.) $40 s$.

$\mathrm{T}$ HIS volume is the second in a series projected by the former Chemical-Biological Co-ordination Center, having as its object the publication of "a manual of test methods dealing with the effects of chemicals on insects".

The first volume has already been reviewed in Nature $(183,352 ; 1959)$. The second volume begins with a group of three papers : "Pre-test Conditions which affect Insect Reaction to Insectieides", "Factors affecting Insects during Exposure to Insecticides", and "Post-exposure Conditions and Determination of Find Point". This division looks orderly on paper, but it is difficult to maintain, and a single chapter would probably have been preferable. Much of the second chapter would be more correctly entitled "Factors affecting Insecticides during the Exposure of Insects"--which is quite a different subject.

The remaining chapters deal with testing methods for insecticides used for specific purposes. Chapter 4, "Exposure to Treated Food Medium", and Chapter 5, "Screening Space Sprays against Flies and Cockroaches", give good straightforward outlines of the methods used, but some comments on the fundamental factors concerned would have assisted the intelligent use of these methods. Chapter 6 deals with "Soil Insecticides". Chapter 7, "Testing Resistance of Chemically Treated Textiles", unlike Chapter 5 , gives full details for carrying out the tests, and it may be said again that it seems regrettable that the 